\title{
Antinucleon spectra in the Dirac equation with scalar and vector Wood-Saxon potentials
}

\author{
R. Lisboa and M. Malheiro*, P. Alberto ${ }^{\dagger}$ and A. S. de Castro** \\ ${ }^{*}$ Instituto Tecnológico de Aeronáutica, CTA, São José dos Campos, São Paulo 12-228-900, Brazil. \\ ${ }^{\dagger}$ Departamento de Física e Centro de Física Computacional, Universidade de Coimbra, \\ P-3004-516 Coimbra, Portugal \\ ${ }^{*+}$ Departamento de Fisica e Quimica, Universidade Estadual Paulista, 12516-410 Guaratinguetá, \\ São Paulo, Brazil
}

\begin{abstract}
We analyze here the spin and pseudospin symmetry for the antinucleon spectra solving the Dirac equation with scalar and vector Wood-Saxon potentials. In relativistic nuclear mean field theories where these potentials have large magnitudes and opposite signs we show that contrary to the nucleon case where pseudospin interaction is never very small and cannot be treated perturbatively, for antinucleon systems this interaction is perturbative and an exact pseudospin symmetry is possible. This result manifests the relativistic nature of the nuclear pseudospin symmetry.
\end{abstract}

Keywords: pseudospin symmetry, wood-saxon potentials, antinucleon

PACS: $21.10 . \mathrm{Hw}, 21.10 . \mathrm{Pc}, 25.43 .+\mathrm{t}, 21.60 . \mathrm{Cs}$

\section{INTRODUCTION}

The pseudospin symmetry is exact when the nucleon doublets $(n, l, j=l+1 / 2)$ and $(n-1, l+2, j=l+3 / 2)$ are degenerate and can therefore be relabeled as pseudospin doublets $(\tilde{n}=n-1, \tilde{l}=l+1, \tilde{j}=\tilde{l} \pm 1 / 2)$. The number $\tilde{l}=l+1$ is the pseudo orbital angular momentum of the lower component of the Dirac spinor [1]. These partners are degenerate when the strong attractive scalar $S$ and repulsive vector $V$ potentials have the same magnitude but opposite signs, $\Sigma=V+S=0$ or at least when $\Sigma^{\prime}=0[2,3]$. The $\Sigma$ potential is responsible for the pseudospin interaction in the second-order differential equation of the lower Dirac spinor. This potential also acts as the binding potential, and cannot be set to zero in nuclei, since in that case there would not be any fermion bound states. By the charge conjugation operation, the sign of vector potential $V$ and energy $E$ are changed while the sign of scalar potential $S$ remains the same [4]. This means that $\Sigma=V+S$ turn into $-\Delta$ and $\Delta=V-S$ into $-\Sigma$. Thus in the case of antinucleons the spin and pseudospin symmetries are realized on the lower and upper Dirac components, respectively. For antifermions the binding potential $-\Delta$ cannot vanish in order to have bound states. Thus, the exact spin symmetry for antinucleons is never possible.

The coupled first-order radial Dirac equations for fermions (left) and antifermions (right) are

$$
\begin{array}{ll}
G_{\kappa}^{\prime}+\frac{1+\kappa}{r} G_{\kappa}=(\varepsilon+2 m-\Delta) F_{K} & \bar{G}_{\bar{\kappa}}^{\prime}+\frac{1+\bar{\kappa}}{r} \bar{G}_{\bar{\kappa}}=\left(\varepsilon_{c}+2 m+\Sigma\right) \bar{F}_{\bar{\kappa}} \\
F_{K}^{\prime}+\frac{1-\kappa}{r} F_{\kappa}=-(\varepsilon-\Sigma) G_{\kappa} & \bar{F}_{\bar{\kappa}}^{\prime}+\frac{1-\bar{\kappa}}{r} \bar{F}_{\bar{\kappa}}=-\left(\varepsilon_{c}+\Delta\right) \bar{G}_{\bar{K}} .
\end{array}
$$

These two sets of equations are very similar to one another if one takes into account the change of the role of $\Delta$ and $\Sigma$ potentials with respect to the upper and lower radial equations. Another important difference comes from the binding energy which is $\varepsilon=E-m$ for fermions while for antifermions it is $\varepsilon_{c}=-E-m$. We solved the Eqs.(1) with Wood-Saxon potentials, where the parameters used are obtained from the best fit of $\mathrm{Pb}^{208}$ experimental neutron spectrum $[5,6]$. In Fig. 1 the strength of scalar potential 

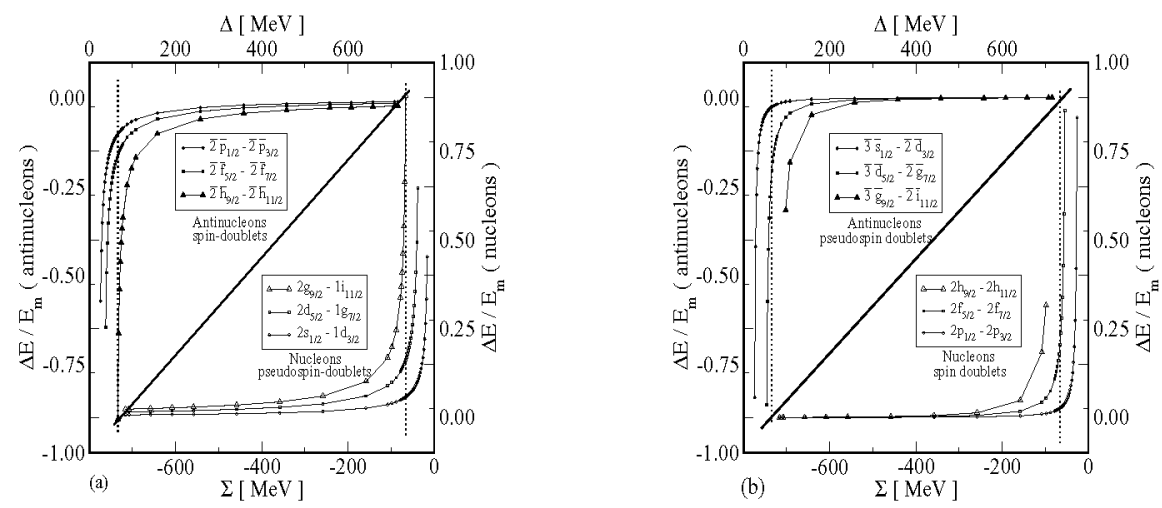

FIGURE 1. The $\Delta E / E_{m}$ versus $\Sigma$ potential for nucleons in bottom side of the diagonal for different (a) pseudospin and (b) spin doublets. The $\Delta E / E_{m}$ versus $\Delta$ potential for antinucleons in upper side of the diagonal for different (a) spin and (b) pseudospin doublets. In Fig.1 the dotted vertical line stands for the $\Sigma_{0}$ and $\Delta_{0}$ values used in ${ }^{208} \mathrm{~Pb}$ calculation.

is $S_{0}=-358 \mathrm{MeV}$ while the vector potential changes in the range $0 \leq\left|V_{0}\right| \leq 292 \mathrm{MeV}$. For nucleons, as $\Sigma$ is the binding potential, the exact pseudospin symmetry cannot be achieved since $\Sigma=0$ would mean that no bound states would exist. In the lower side of the diagonal in the Fig.1(a) the pseudospin interaction is small when $\Sigma \rightarrow 0$ but cannot be treated perturbatively because $\Delta E / E_{m}$ is large. When $|\Sigma|$ is large the fermions are more bounded $\left(E_{m}\right.$ large) and relativistic so that $\Delta E / E_{m}$ is small. In the lower side of the diagonal in the Fig.1(b) the spin symmetry is not good near the dotted vertical line (nuclear physics values) because $\Delta$ is big. When $\Delta \rightarrow 0$ spin symmetry is exact for fermions. Because of the inversion of the role played by $\Sigma$ and $\Delta$ potentials for antifermions ( $\Delta$ is the now the binding potential) exact spin symmetry cannot be reached because one cannot set $\Delta=0$. Actually, in the upper side of the diagonal in the Fig.1(a) we see that $\Delta E / E_{m}$ is large when $\Delta \rightarrow 0$. Furthermore, we see from the upper side of the diagonal in the Fig.1(b) that the case $\Sigma=0$ is possible and we have an exact pseudospin symmetry with degenerate antinucleon pseudospin partners $(\Delta E=0)$.

Summarizing, in the nucleus the charge conjugation transformation relates the spin symmetry of the negative energy bound state solutions (antinucleons) to the pseudospin symmetry of the positive energy bound state solutions (nucleons). We show that for antinucleon systems the exact pseudospin can be realized $(\Sigma=0)$ while still having bound states, since $\Delta$ is the binding potential. This fact manifests the relativistic nature of the nuclear pseudospin symmetry.

\section{ACKNOWLEDGMENTS}

This work was supported by FAPESP, CAPES-GRICES/183/07, CNPq, and POCI/FP/81923/2007 scientific programs .

\section{REFERENCES}

1. J. N. Ginocchio, Phys. Rev. Lett. 78, 436 (1997); Phys. Rep. 414, 165 (2005).

2. J. Meng, K. Sugawara-Tanabe, S. Yamaji, P. Ring, and A. Arima, Phys. Rev. C 58, R628 (1998).

3. J. Meng, K. Sugawara-Tanabe, S. Yamaji, and A. Arima, Phys. Rev. C 59, 154 (1999).

4. A. S. de Castro, P. Alberto, R. Lisboa and M. Malheiro, Phys. Rev. C 73, 054309 (2006).

5. P. Alberto, M. Fiolhais, M. Malheiro, A. Delfino, and M. Chiapparini, Phys. Rev. C 65, 034307 (2002).

6. P. Alberto, M. Fiolhais, M. Malheiro, A. Delfino and M. Chiapparini, Phys. Rev. Lett. 86, 5015 (2001). 
Copyright of AIP Conference Proceedings is the property of American Institute of Physics and its content may not be copied or emailed to multiple sites or posted to a listserv without the copyright holder's express written permission. However, users may print, download, or email articles for individual use. 